\title{
Boosting weakened synapses to treat Alzheimer's disease
}

\author{
Robert J. McDonald ${ }^{1} \cdot$ Scott H. Deibel $^{1}$
}

Published online: 7 December 2016

(C) Psychonomic Society, Inc. 2016

\begin{abstract}
Summary This paper highlights a recent report by Roy and colleagues showing that boosting plasticity in synapses activated during initial memory encoding ameliorates memory impairments found in the early stages of the familial version of Alzheimer's disease. Our goal was to describe the main features of the report and evaluate the approach and implications of the work.
\end{abstract}

Keywords Memory $\cdot$ Engram $\cdot$ Alzheimer's disease · Plasticity $\cdot$ Genetics

\section{Introduction}

Alzheimer's disease (AD) is often not diagnosed until its late stages, which is predominately characterized by neurodegeneration. Treatments are largely ineffective for $\mathrm{AD}$, and one explanation is that once the disease is recognized pathology is irreversible. Thus, recognizing and characterizing early $\mathrm{AD}$ is of paramount importance (Gidyk, Deibel, Hong, \& McDonald, 2015).

Roy and colleagues (2016) recently investigated the mechanisms underlying memory in a mouse model of early $\mathrm{AD}$. The authors concluded that the memory impairment in early $\mathrm{AD}$ is due to an inability to retrieve the memory and targeted stimulation of the hippocampal (HPC) cells involved in a specific memory to facilitate natural retrieval of that memory. A transgenic mouse model of $\mathrm{AD}$ was used that only displayed amyloid

Robert J. McDonald

r.mcdonald@uleth.ca

1 Department of Neuroscience, Canadian Centre for Behavioural Neuroscience, University of Lethbridge, 4401 University Drive, Lethbridge, AB, Canada T1K 3M4 plaques in the dentate gyrus (DG) region of the HPC and medial entorhinal cortex at 9 and not 7 months of age. While both ages had impaired long-term memory (LTM; 24-h) of single context fear conditioning (CFC), only 7-month-old animals had normal short-term memory (STM; 1-h). Interestingly, although the density of DG cells was normal in the 7-month-old mice, these animals had decreased cFos (a marker of neuronal activity)-labeled DG cells compared to control mice after the LTM test. The authors concluded that the 7-month-old animals represented early $\mathrm{AD}$ (henceforth referred to as early $\mathrm{AD}$ mice).

The authors have previously reported that a population of DG cells representing a specific memory (engram) can be identified via transgenic modifications and then manipulated with optogenetics (Liu et al., 2012). In the current study, the authors created early engram AD mice that also had impaired LTM. Amazingly, when the DG engram was optogenetically activated in a novel context these mice displayed freezing behavior similar to control animals. The authors replicated this effect in several different transgenic mouse models. The authors concluded that the LTM impairment in these early AD mice was due to failure to retrieve the memory.

Next the authors delved deeper into the nature of the engram cells. While neurogenesis and functional connectivity of the DG engram cells was normal, only the DG cells that were part of the engram had decreased dendritic spine density. The authors hypothesized that induction of long-term potentiation (LTP) in the DG engram cells would restore spine density and thus rescue memory. LTP was induced in the DG engram cells by optogenetically stimulating entorhinal cortex perforant path cells that terminated in these DG cells. Memory recall was tested 48 -h after this manipulation without any optogenetic manipulations. This procedure restored spine density of DG engram cells and rescued the fear memory (replicated for other types of memory) for up to 6 days after the optical LTP induction. This effect was dependent on inducing LTP in only the engram cells, as 
induction of LTP in a large population of perforant path terminals in the DG did not rescue memory. To ensure that the induction of LTP in the DG engram cells was not inducing LTP in other DG cells and thus influencing memory retrieval, they developed an engram-specific virus that would only ablate the DG engram cells. Ablation of the DG engram cells after induction of LTP impaired memory. These data suggest that LTP induction in only the DG engram cells facilitates natural memory retrieval. The authors conclude that, in contrast to previous reports, the memory impairment in early $\mathrm{AD}$ is due to a failure to retrieve encoded information rather than an inability to encode that information.

There is a significant amount of excitement around this work, particularly from a technical point of view, but the work also has conceptual, theoretical, and applied implications. Our goal is to provide some alternative views of the work and place it in the context of the field.

\section{Validity and relevance of animal models of AD}

We and others have noted that most of the animal models of $\mathrm{AD}$ are at best relevant for research trying to understand and develop treatments for the rare familial version of AD. Ninety percent of $\mathrm{AD}$ patients have the sporadic version (SAD) and the cause of this version of the disease is unknown. Accordingly, no good animal models of SAD currently exist, although we have developed a conceptual framework and examples of what these kinds of models might look like. Our basic idea is that the etiology of $\mathrm{SAD}$ is complex and heterogeneous, manifesting itself when the presence of various combinations of co-factors coexist (Craig, Hong, \& McDonald, 2011; Gidyk et al., 2015). Proof of principle for this etiological theory comes from experiments showing that different combinations of co-factors linked to dementia produce HPC dysfunction and learning and memory impairments in rodents. The pattern of results from this work is revealing with regard to SAD etiology because some combinations produced neurodegeneration while others impaired plasticity. We have argued that multiple mechanisms can be altered depending on the combinations present in an individual, and plasticity would be a treatment target in some but not others (Craig et al., 2011; Gidyk et al., 2015). The memory rescue approach proposed in the Roy paper might be more effective for the SAD population, particularly those patients with plasticity-based impairments, but this remains to be seen. A strong push to develop valid animal models of SAD must be a priority for the $\mathrm{AD}$ research community (Gidyk et al., 2015).

\section{What is being rescued?}

Another issue going forward is which memories for an individual with $\mathrm{AD}$ could be rescued? Would only recent events, like those manipulated in the Roy paper, be potentially brought back, and for how long? One key aspect of human memory that is impacted in $\mathrm{AD}$ is the ability to recall memories that constitute our personal history and that have accumulated over decades. The current method has only purportedly demonstrated that retrieval of a simple and very recently acquired memory can be enhanced.

\section{Murine mouse models: no problems?}

It is always important to appreciate potential problems associated with the transgenic approach. Concerns are repeatedly raised in the literature regarding the use of transgenics for animal models of human disease states like AD including: (i) non-cognitive effects of the genetic manipulation that alter supports for learning and memory functions including sensory, motor, and motivational deficits; (ii) overexpression issues; (iii) the problem of flanking genes; (iv) disease validity; and (v) the background strain problem (Schellinck, Cyr, \& Brown, 2010). The current approach not only uses a transgenic as the foundation for the $\mathrm{AD}$ model but these animals are then crossed with other transgenic mice. Independent verification of the specificity of the deficit in these new types of transgenics is warranted to assuage this concern.

\section{Summary}

In summary, Roy and colleagues' findings are exciting, pushing out boundaries about the nature of the neural basis of learning and memory in the mammalian brain and potential approaches to treating diseases of memory. Because of these potential impacts, each aspect of the work needs to be scrutinized to the highest standard to assess its ultimate contribution.

\section{References}

Craig, L. A., Hong, N. S., \& McDonald, R. J. (2011). Revisiting the cholinergic hypothesis in the development of Alzheimer's disease. Neuroscience and Biobehavioral Reviews, 35(6), 1397-1409. doi:10.1016/j.neubiorev.2011.03.001

Gidyk, D. C., Deibel, S. H., Hong, N. S., \& McDonald, R. J. (2015). Barriers to developing a valid rodent model of Alzheimer's disease: From behavioral analysis to etiological mechanisms. Frontiers in Neuroscience, 9(July), 1-18. doi:10.3389/fnins.2015.00245

Liu, X., Ramirez, S., Pang, P. T., Puryear, C. B., Govindarajan, A., Deisseroth, K., \& Tonegawa, S. (2012). Optogenetic stimulation of a hippocampal engram activates fear memory recall. Nature, 484(7394), 381-385. doi:10.1038/nature11028.Optogenetic

Roy, D. S., Arons, A., Mitchell, T. I., Pignatelli, M., Ryan, T. J., \& Tonegawa, S. (2016). Memory retrieval by activating engram cells in mouse models of early Alzheimer's disease. Nature, 531(7595), 508-512. doi:10.1038/nature17172

Schellinck, H. M., Cyr, D. P., \& Brown, R. E. (2010). How many ways can mouse behavioral experiments go wrong? Confounding variables in mouse models of neurodegenerative diseases and how to control them. Advances in the Study of Behavior, 41, 255-366. 